\title{
Clozapine Interaction with Phosphatidyl Inositol 3-Kinase (PI3K)/Insulin-Signaling Pathway in Caenorhabditis elegans
}

\author{
Rakesh Karmacharya ${ }^{1,2,3}$, Gregory R Sliwoski ${ }^{2}$, Miriam Y Lundy ${ }^{2}$, Raymond F Suckow ${ }^{4}$, Bruce M Cohen ${ }^{1,2}$ \\ and Edgar A Buttner*, I,2 \\ 'Department of Psychiatry, Harvard Medical School, Boston, MA, USA; ${ }^{2}$ Mailman Research Center, McLean Hospital, Belmont, MA, USA; \\ ${ }^{3}$ Chemical Biology Program, Broad Institute of Harvard and MIT, Cambridge, MA, USA; ${ }^{4}$ Department of Analytical Psychopharmacology, \\ New York State Psychiatric Institute, New York, NY, USA
}

\begin{abstract}
Clozapine has superior and unique effects as an antipsychotic agent, but the mediators of these effects are not known. We studied behavioral and developmental effects of clozapine in Caenorhabditis elegans, as a model system to identify previously undiscovered mechanisms of drug action. Clozapine induced early larval arrest, a phenotype that was also seen with the clozapine metabolite $\mathrm{N}$-desmethyl clozapine but not with any other typical or atypical antipsychotic drug tested. Mutations in the insulin receptor/daf-2 and phosphatidyl inositol 3-kinase (PI3K)/age-I suppressed clozapine-induced larval arrest, suggesting that clozapine may activate the insulinsignaling pathway. Consistent with this notion, clozapine also increased the expression of an age- $/:$ :GFP reporter. Activation of the insulin-signaling pathway leads to cytoplasmic localization of the fork head transcription factor FOXO/daf-16. Clozapine produced cytoplasmic localization of DAF-16:: GFP in arrested LI larvae, in contrast to stressors such as starvation or high temperature, which produce nuclear localization of DAF-16:: GFP in arrested LI larvae. Clozapine also inhibited pharyngeal pumping in C. elegans, an effect that may contribute to, but did not explain, clozapine-induced larval arrest. Our findings demonstrate a drug-specific interaction between clozapine and the PI3K/insulin-signaling pathway in C. elegans. As this pathway is conserved across species, the results may have implications for understanding the unique effects of clozapine in humans.

Neuropsychopharmacology (2009) 34, 1968-1978; doi: I0.1038/npp.2009.35; published online 25 March 2009
\end{abstract}

Keywords: clozapine; PI3K; AGE-I; DAF-2; insulin signaling; schizophrenia

\section{INTRODUCTION}

The treatment of patients with schizophrenia has been hindered by the limited efficacy of currently available antipsychotic medications and the frequent and disabling side effects produced by these drugs (Freedman, 2005; Lieberman et al, 2005). Development of improved treatments has been slowed by a poor understanding both of the pathophysiology of psychosis and the mechanisms of action of current medications (Carpenter and Koenig, 2008). Although antagonism of monoamine receptors is a shared property of antipsychotic agents, the drugs vary greatly in relative potency at these receptors, and it is not known how actions at these common targets mediate each drug's specific clinical effects (Arnt and Skarsfeldt, 1998; Gardner et al, 2005).

\footnotetext{
* Correspondence: Dr EA Buttner, Department of Psychiatry, Mailman Research Center, McLean Hospital, II 5 Mill Street, Belmont, MA 02478, USA, Tel: + 617855 2074, Fax: + 617876 5148,

E-mail: nbuttner@gmail.com

Received 6 November 2008; revised 2 February 2009; accepted 18 February 2009
}

Clozapine stands out among the antipsychotic medications for its superior efficacy in treatment-refractory schizophrenia and its production of minimal extrapyramidal symptoms (Kane et al, 1988; Kumra et al, 2008). More than other antipsychotics, clozapine has been shown to reduce negative symptoms and to ameliorate cognitive deficits in patients with schizophrenia (Brar et al, 1997; Lindenmayer et al, 1998; Meltzer and McGurk, 1999). The use of clozapine is limited by its occasional production of agranulocytosis, a complication that requires frequent monitoring of white blood cells (Krupp and Barnes, 1992). In addition, there is a significant drug-related incidence of new-onset diabetes mellitus, as well as dyslipidemia and weight gain (Lamberti et al, 2006). The molecular basis of both clozapine's unique superior efficacy and its side effects are unknown, although various mechanisms have been proposed (Brunello et al, 1995).

C. elegans provides a versatile system that can be used to identify targets and pathways affected by psychoactive drugs (Weinshenker et al, 1996; Bettinger et al, 2004). There are many genetic pathways originally characterized in C. elegans that have generalized to mammalian biology, such as the control of vulval development by the ras pathway (Horvitz and Sternberg, 1991), control of apoptosis 
by the programmed cell death pathway (Hengartner and Horvitz, 1994), RNA interference (Fire et al, 1998), and the control of development by microRNAs (Ambros, 2001; Ruvkun, 2001). Many gene systems of interest in the study of psychiatric neurobiology are conserved between $C$. elegans and mammals, such as neurotransmitter receptors, including G-protein-coupled receptors and ligand-gated channels, second-messenger systems, many ion channels, synaptic proteins, and enzymes involved in neurotransmitter biosynthesis and metabolism (Bargmann, 1998). Largescale genetic screens in invertebrates allow identification of genetic targets without prior knowledge of the role of such targets, an approach not possible in mouse models (Riddle et al, 1997; Sieburth et al, 2005). Human homologs of C. elegans genes may point to biological mechanisms that provide insight into clinically relevant mechanisms in humans. For example, studies of development and programmed cell death in $C$. elegans have led to the identification of genes that are conserved in mammals, and that, when mutated, cause neurodegenerative disease and cancer in humans (Horvitz, 1999).

We undertook a study in $C$. elegans to identify novel mechanisms underlying the effects of clozapine. Similar studies in $C$. elegans have led to the identification of novel targets of psychoactive agents such as the antidepressant fluoxetine (Choy and Thomas, 1999; Choy et al, 2006) as well as the identification of the calcium-activated large conductance BK potassium channel as a target of ethanol (Davies et al, 2003). We observed a clozapine-specific developmental phenotype resulting in early larval arrest. We report that age-1 mutants suppress clozapine-induced larval arrest, indicating interactions between clozapine and the PI-3 kinase (PI3K)/insulin-signaling pathway. This pathway mediates a diverse array of biological processes in C. elegans, such as metabolism, growth, and longevity. The pathway, which is well-conserved between C. elegans and humans, includes homologs of the insulin receptor/daf-2, $\mathrm{PI} 3 \mathrm{~K} /$ age-1, the serine-threonine kinases Akt/akt-1 and akt2 and $\mathrm{PDK} 1 / p d k-1$, and the fork head transcription factor FOXO/daf-16. DAF-16 is a major target of the pathway and is antagonized by activation of DAF-2 (Paradis and Ruvkun, 1998; Pierce et al, 2001).

\section{MATERIALS AND METHODS}

C. elegans strains were obtained from the Caenorhabditis Genetics Center (CGC) in Minneapolis, MN. Experiments were conducted with the $C$. elegans Bristol strain N2 as the wild-type parent of our mutant strains. Nematodes were grown under standard culture conditions at $20^{\circ} \mathrm{C}$. The following strains were used: CB1112 cat-2(e1112), MT7988 bas-1(ad446), MT9455 tph-1(n3722), GR1321 tph-1(mg280), CB1141 cat-4 (e1141), JT603 gpl-2 (sa603), JT734 goa-1 (sa734), MT2605 unc-43 (n498n1186), TJ1052 age-1(hx546), TJ412 age-1(hx542); fer-15(b26), CB1370 daf-2(e1370), DR1564 daf-2(m41), DR1567 daf-2(m577), DR1568 daf2(e1371), GR1318 pdk-1(mg142), JT709 pdk-1(sa709), JT9609 pdk-1(sa680), DR26 daf-16(m26), CZ1197 Punc25::GFP(juIs73), TJ356 zIs356[DAF-16::GFP], BC10837 [dpy-5(e907); sEx10837 (age-1::GFP)] (McKay et al, 2003), FK229 egl-4(ks61), DA695 egl-19(ad695), PR678 tax-4(p678), PR694 tax-2(p694), DR2281 daf-9(m540), DR47 daf-11(m47), DR62 daf-7(m63), DR63 daf-4(m63). The sEx10837 (age-1::GFP) array was integrated in the following manner: 100 animals were irradiated with $\mathrm{X}$-ray on 20 medium plates, allowed to starve and transferred to fresh plates. After $24 \mathrm{~h}, 300$ transgenic animals were picked to fresh plates and scored for presence of fluorescence in the progeny. The $t b h-1$ mutant allele $n 3722$ and $t p h-1$ mutant allele $m g 280$ were gifts from Dr Mark Alkema (University of Massachusetts, Worcester, MA). We obtained clozapine, perphenazine, and haloperidol from Sigma-Aldrich, $\mathrm{N}$ desmethyl clozapine from Tocris, and olanzapine from Waterstone Technology. Nematodes were grown and studied under standard culture conditions at $20^{\circ} \mathrm{C}$ unless otherwise noted.

\section{Larval Arrest}

Drug plates were prepared by pipetting $100 \mu$ of stock solution in $100 \%$ ethanol onto the agar surrounding the bacterial lawn. 10 gravid adults were placed in two seeded Nematode Growth Medium (NGM) plates each with $1 \%$ ethanol and either $200 \mu \mathrm{g} / \mathrm{ml}$ clozapine, $50 \mu \mathrm{g} / \mathrm{ml}$ haloperidol, or $100 \mu \mathrm{g} / \mathrm{ml}$ olanzapine. Gravid adults were removed after $24 \mathrm{~h}$. Numbers of nematodes in different stages were counted every day. The smallest larvae on the plates were counted as L1 whereas larger larvae that lacked a pre-vulva space were scored as L2. L3 larvae were scored using the following identifying factors: they are larger than L2, they have a pre-vulval space, and they exhibit much greater locomotion than L1 or L2 larvae. Dead worms and unhatched eggs were not counted. To examine the effects of clozapine on different larval stages, freshly laid eggs from gravid adults were incubated at $20^{\circ} \mathrm{C}$. Larvae of different stages (L1, L2, L3, and L4) were picked at specific time intervals corresponding to the developmental stage at $20^{\circ} \mathrm{C}$ and placed on fresh plates containing $200 \mu \mathrm{g} / \mathrm{ml}$ clozapine. Plates were incubated at $20^{\circ} \mathrm{C}$ and worms of different larval stages counted for the next 12 days or until all worms had reached adulthood.

\section{Measurement of Tissue Clozapine Level}

Eggs from bleached worms were washed and placed on 20 medium NGM plates containing $200 \mu \mathrm{g} / \mathrm{ml}$ of clozapine. After $48 \mathrm{~h}$ at $20^{\circ} \mathrm{C}$, arrested larvae were collected. They were washed and resuspended in $1 \mathrm{ml}$ of $\mathrm{M} 9$ buffer and subjected to four freeze-thaws. This solution was vortex-mixed and an aliquot immediately sampled for analysis. The homogenate was extracted by liquid-liquid extraction followed by back extraction of the organic phase into phosphate buffer and an aliquot of this aqueous extract was directly injected into the HPLC system. Clozapine and $N$-desmethyl clozapine levels were determined through HPLC with UV detection.

\section{Dose Response}

Experiments were set up with two plates per condition and 10 adults per plate on day 1 . Concentrations of drugs used were in the following range: $50-200 \mu \mathrm{g} / \mathrm{ml}$ for clozapine and $\mathrm{N}$-desmethyl clozapine, $10-50 \mu \mathrm{g} / \mathrm{ml}$ for haloperidol, and $10-100 \mu \mathrm{g} / \mathrm{ml}$ for olanzapine. Plates were scored on days 3 , 
6, 9, and 12. Any plate that had grown beyond one generation was no longer scored.

\section{DAF-16 Localization}

Adult TJ356 worms containing the integrated daf-16::gfp marker $z I s 356$ were placed on plates with $200 \mu \mathrm{g} / \mathrm{ml}$ clozapine or $1 \%$ ethanol and removed after $24 \mathrm{~h}$. After another $48 \mathrm{~h}$ at $20^{\circ} \mathrm{C}$, the arrested larvae were put on slides using tricaine and tetramisol to anesthetize them and worms were scored for nuclear localization of GFP. To obtain starved larvae, eggs from bleached worms were placed on plates without food. The hatched starved larvae were collected after $48 \mathrm{~h}$ and put on slides using tricaine and tetramisol to anesthetize them. For all three conditions, worms were scored within $5 \mathrm{~min}$ of being put on the slide, because paralysis induced DAF-16 nuclear localization after $20 \mathrm{~min}$ in control worms. Photographs were taken with an Axio Image A1 microscope and an AxioCAM MRM camera.

\section{Pharyngeal Pumping}

Adult worms were placed overnight on plates with $1 \%$ ethanol or $200 \mu \mathrm{g} / \mathrm{ml}$ clozapine and removed the following morning. Pharyngeal pumping of the hatched larvae was scored. Five larvae at a time were transferred from each plate onto fresh plates with abundant food lacking any drugs or alcohol. The worms were allowed to settle for $5 \mathrm{~min}$ and the number of pumps was counted during a 30-s time frame. Only worms on the bacterial lawn were scored.

\section{Induction Studies}

For the age-1::GFP worms, the heterozygotes are wild-type and the worms missing the array are dumpy. So, only the wild-type adults were chosen. Ten adult worms were placed on three plates each of $1 \%$ ethanol and $200 \mu \mathrm{g} / \mathrm{ml}$ clozapine and removed after $18 \mathrm{~h}$. After six hours, hatched worms were put on slides using tricaine and tetramisol to anesthetize them and GFP expression was scored. The number of GFP-positive cells in the intestine was then scored. GFP expression was noted for each section as being none, dull, moderate, or bright.

\section{RESULTS}

\section{Clozapine Induces Early Larval Arrest in C. elegans}

We allowed adult worms to lay eggs on plates containing clozapine, the typical antipsychotics haloperidol and perphenazine, or the atypical antipsychotic olanzapine, which is structurally related to clozapine. We then studied the development of hatched larvae over time. The presence of the vehicle, $1 \%$ ethanol alone, slowed the development of control animals slightly, as expected (Figure 1h) (Lee and McIntire, 2006; Davis et al, 2008). Wild-type worms in the presence of $1 \%$ ethanol took 4 days to reach adulthood from the time the eggs were laid. Worms exposed to haloperidol, perphenazine and olanzapine progressed through development to adulthood with a very slight delay, taking 4-5 days to reach adulthood (Figure la, d, e, f, and $\mathrm{h}$ ). Besides this delay, no other changes were observed in the growth cycle. However, newly hatched larvae exposed to clozapine did not develop beyond the early larval stages even after 2 weeks (Figure $1 \mathrm{~b}$ and $\mathrm{h}$ ). Worms arrested in the presence of clozapine were primarily in the L1 stage with only $18 \%$ of arrested worms reaching the L2 stage and no worms of later stages were present.

We quantified the effects of clozapine on the development of C. elegans by performing dose-response and time-course studies. Larval arrest with exposure to clozapine was dosedependent. By day 12, N2 larvae exposed to $125 \mu \mathrm{g} / \mathrm{ml}$ clozapine had developed on to adulthood. N2 larvae exposed to $150 \mu \mathrm{g} / \mathrm{ml}$ clozapine had intermediate results with some worms reaching the L3 larval stage, but no larvae developing into adults (Figure 2). There was complete L1L2 larval arrest with exposure to clozapine concentrations of $175 \mu \mathrm{g} / \mathrm{ml}$ or greater (Figure 2). We also tested the effect of the clozapine metabolite $N$-desmethyl clozapine on larval development, because this metabolite has been hypothesized to play a major role in the therapeutic effects of clozapine (Sur et al, 2003; Weiner et al, 2004). We observed larval arrest with $N$-desmethyl clozapine at $200 \mu \mathrm{g} / \mathrm{ml}$ (Figure 1c). Exposure to the highest soluble level we could achieve with haloperidol in $1 \%$ ethanol $(50 \mu \mathrm{g} / \mathrm{ml})$ did not result in larval arrest (Figure $1 \mathrm{~d}$ and $\mathrm{h}$ ). Clinically, haloperidol is used at doses more than an order of magnitude lower than clozapine. Nonetheless, to achieve concentrations similar to that of clozapine, we also conducted studies with $200 \mu \mathrm{g} / \mathrm{ml}$ haloperidol, although this necessitated a four-fold higher final concentration of ethanol for solubility. No larval arrest was seen with haloperidol in this condition. We conducted similar studies with the atypical antipsychotic drug olanzapine, which, among the available antipsychotic drugs, is structurally most similar to clozapine, and with the typical antipsychotic drug perphenazine at similar concentrations. We did not observe larval arrest with either of those widely used antipsychotics (Figure 1e, f, and h).

These larval arrest studies were performed with clozapine exposure initiated in the embryonic state. To study the nature of the larval arrest further, we examined whether clozapine-induced larval arrest resulted only from exposure prior to the L1 stage or whether developmental arrest could be induced in later larval stages as well. Larvae were staged based on known growth rates at $20^{\circ} \mathrm{C}$ and physical

\footnotetext{
Figure I Clozapine induces larval arrest in C. elegans. Representative photographs of larval development, at $\times 30$ magnification (a-g). (a) N2 worms exposed to $1 \%$ ethanol for 4 days. Adult worms and their eggs are seen. (b) N2 worms exposed to $200 \mu \mathrm{g} / \mathrm{ml}$ clozapine for 10 days. All worms are arrested at LI-L2 stages. (c) $\mathrm{N} 2$ worms exposed to $200 \mu \mathrm{g} / \mathrm{ml} \mathrm{N}$-desmethyl clozapine for 10 days. All worms are arrested at LI-L2 stages. (d) N2 worms exposed to $50 \mu \mathrm{g} / \mathrm{ml}$ haloperidol for 5 days. Adult worms and eggs are seen. (e) N2 worms exposed to $200 \mu \mathrm{g} / \mathrm{ml}$ olanzapine for 5 days. Adult worms and eggs are seen. (f) N2 worms exposed to $200 \mu \mathrm{g} / \mathrm{ml}$ perphenazine for 5 days. Adult worms are seen. (g) age-l (hx546) worms exposed to $200 \mu \mathrm{g} / \mathrm{ml}$ clozapine for 7 days. Worms are no longer arrested in the larval stages, and adult worms are seen. (h) Time course of development for N2 worms with exposure to $1 \%$ ethanol, haloperidol, perphenazine, olanzapine or clozapine. Abbreviations: $Y A=$ young adult; $G A=$ gravid adult.
} 
characteristics. We exposed separate populations of L1, L2, L3, and L4 larvae to clozapine (Supplementary Figure 1). Exposing L1 larvae to clozapine resulted in complete larval arrest. Most L1 larvae exposed to clozapine arrested development at the L1 stage, although a few progressed to the L2 stage and then either arrested development or died.
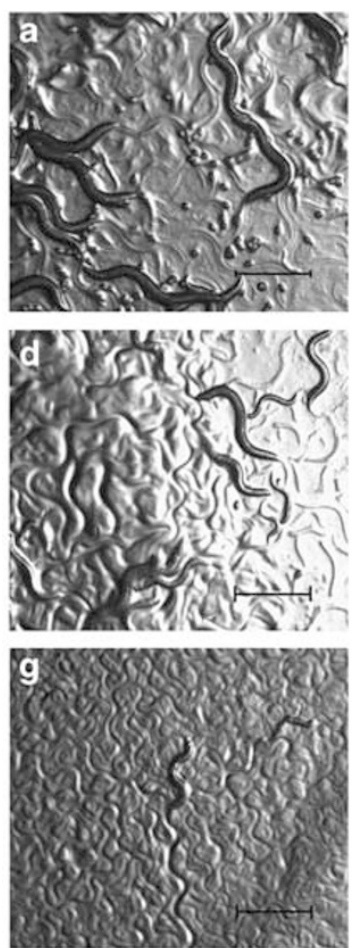
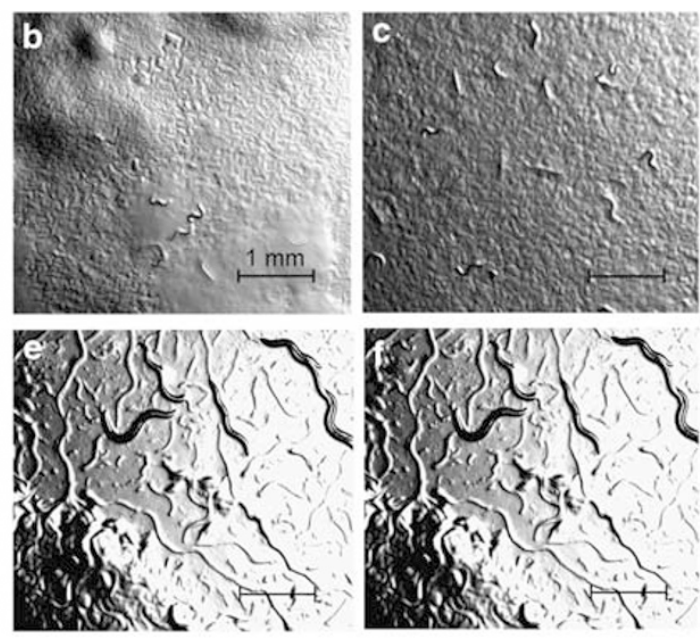

h

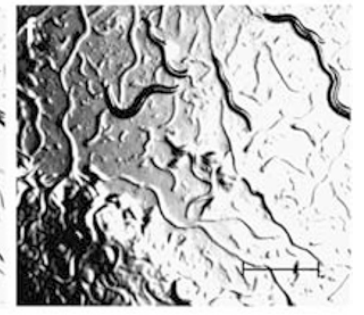

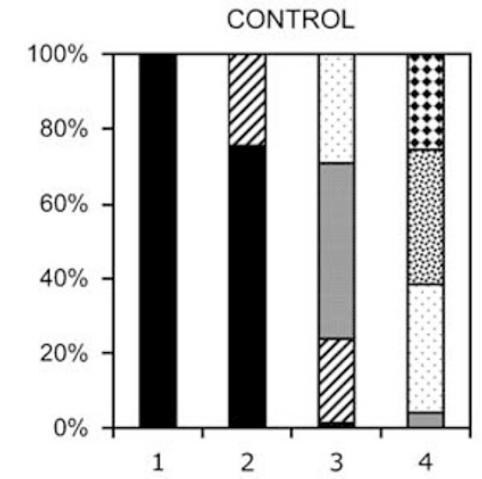
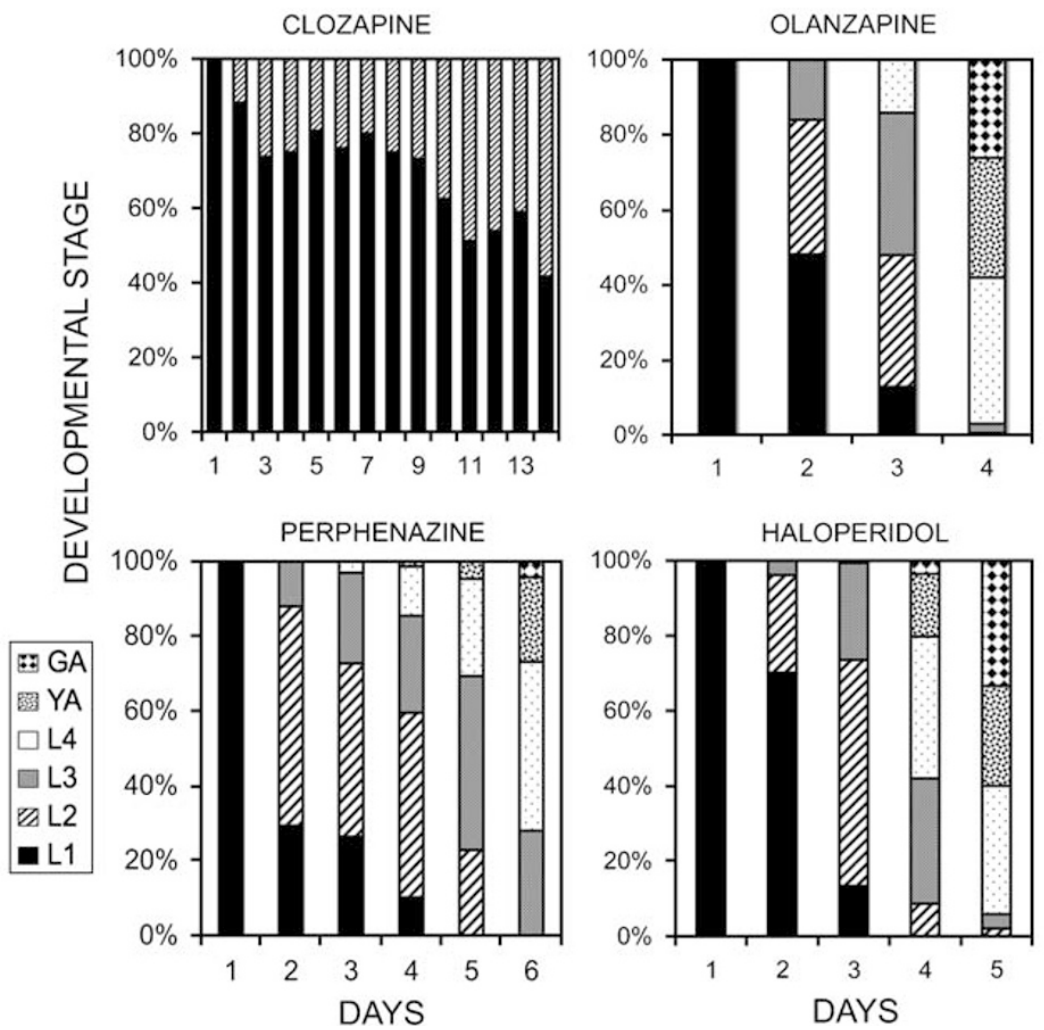

Neuropsychopharmacology 
We observed a significantly higher mortality rate in L2 larvae exposed to clozapine than those exposed as eggs or as L1 larvae, but they did not arrest. L3 larvae exposed to clozapine did not undergo developmental arrest, although their growth was delayed and many of these worms died as young adults. The worms that did manage to reach gravid adulthood became bags of worms. L4 larvae exposed to clozapine developed to gravid adulthood and successfully laid eggs. In summary, clozapine-induced arrest occurs primarily at the L1 stage. Once a worm enters the L3 larval stage, clozapine exposure slows the rate of development and increases mortality but does not cause complete arrest.

We determined the tissue levels of clozapine and its active metabolite $\mathrm{N}$-desmethyl clozapine using HPLC to compare the drug levels in the worms with tissue levels observed in patients. We found that worms exposed to $200 \mu \mathrm{g} / \mathrm{ml}$ of clozapine had total clozapine and $N$-desmethyl clozapine concentrations of $18.02 \mu \mathrm{g} / \mathrm{ml}(17.2 \mu \mathrm{g} / \mathrm{ml}$ clozapine and $0.82 \mu \mathrm{g} / \mathrm{ml}$ of $\mathrm{N}$-desmethyl clozapine). Average serum levels of clozapine and $\mathrm{N}$-desmethyl clozapine in patients taking clozapine have been reported to be $460 \pm 245 \mathrm{ng} / \mathrm{ml}$, with average clozapine levels of $265 \pm 143 \mathrm{ng} / \mathrm{ml}$ and $N$-desmethyl clozapine levels of $194 \pm 117 \mathrm{ng} / \mathrm{ml}$ (Centorrino et al, 1996). It should be noted that these are serum levels, and the brain levels of clozapine and $\mathrm{N}$-desmethyl clozapine are expected to be higher, given the lipophilic properties of clozapine. Measurements of serum and brain levels of clozapine in rats have shown that the brain and serum levels are closely correlated, but there is a 24-fold higher concentration of clozapine in the brain (Baldessarini et al, 1993). Hence, the levels that one would expect in human brains based on these studies $(\sim 11 \mu \mathrm{g} / \mathrm{ml})$ is very close to the levels that we found in the worm tissue $(18.02 \mu \mathrm{g} / \mathrm{ml})$.

\section{age-1 Mutants Suppress Clozapine-induced Larval Arrest}

Given the putative roles of dopaminergic and serotonergic systems in the pathogenesis of schizophrenia and known interactions between clozapine and these neurotransmitter systems, we initially studied worms deficient in dopamine and/or serotonin to see if such mutants suppressed clozapine-induced larval arrest (Gardner et al, 2005). We tested the mutants cat-2(e1112), which has a mutation in the dopamine biosynthetic enzyme tyrosine hydroxylase, tph-1 (mg280), which has a mutation in the serotonin biosynthetic enzyme tryptophan hydroxylase, bas-1(ad446), which has a mutation in the serotonin and dopamine biosynthetic enzyme aromatic amino acid decarboxylase, and cat-4 (e1141), which has a mutation in the serotonin and dopamine biosynthetic enzyme GTP cyclohydrolase I. Hundred per cent of these mutant worms were arrested in the L1-L2 stage in the presence of clozapine. We then examined worms with mutations in various other neurotransmitter and signal transduction systems to find mutants that suppressed clozapine-induced larval arrest.

Age-1(hx546) worms, which contain a mutation in phosphatidyl inositol 3-kinase (PI3K), suppressed clozapine-induced larval arrest (Figures $1 \mathrm{~g}$ and 2 ). In the absence of the antipsychotics, these mutants grow to adulthood along the same time course as N2 worms (Supplementary Figure 2). A delay in growth was observed in age-1 worms exposed to clozapine, but these mutants developed through all larval stages into adulthood and laid eggs (Figures $1 \mathrm{~g}$ and 2). This effect seen with age-1 was dose-dependent (Figure 2). For example, on exposure to a lower concentration of clozapine $(100 \mu \mathrm{g} / \mathrm{ml})$, about $60 \%$ of $\mathrm{N} 2$ worms were L1 or L2 on day 12, whereas all animals had reached adulthood on the age-1 plates. To confirm the involvement of these genes in suppression of clozapine-induced larval arrest, we studied another available age-1 allele $h \times 542$ and found similar suppression of larval arrest (Supplementary Figure 3). We also found that daf-2(e1370) animals, which contain a mutation in the insulin/IGF-1 receptor, partially suppressed clozapine-induced larval arrest, growing beyond the early larval stages, but not to adulthood at $200 \mu \mathrm{g} / \mathrm{ml}$ clozapine (Figure 2). Similar results were seen with three other daf-2 alleles (e1370, $m 41, m 577)$ (Supplementary Figure 3).

C. elegans larvae can enter an alternative dauer developmental stage during unfavorable environmental conditions (Riddle et al, 1997). We considered the possibility that clozapine could be forcing the worms to enter the dauer stage. Dauer worms can survive harsh conditions and live for extended periods of time. Dauer larvae can be recovered by resistance to treatment with $1 \%$ SDS (sodium dodecyl sulfate). We treated the clozapine-arrested worms with $1 \%$ SDS and found that none of the worms had entered dauer stage. Dauer arrest is affected by multiple pathways, including the insulin-signaling pathway, the TGF- $\beta$

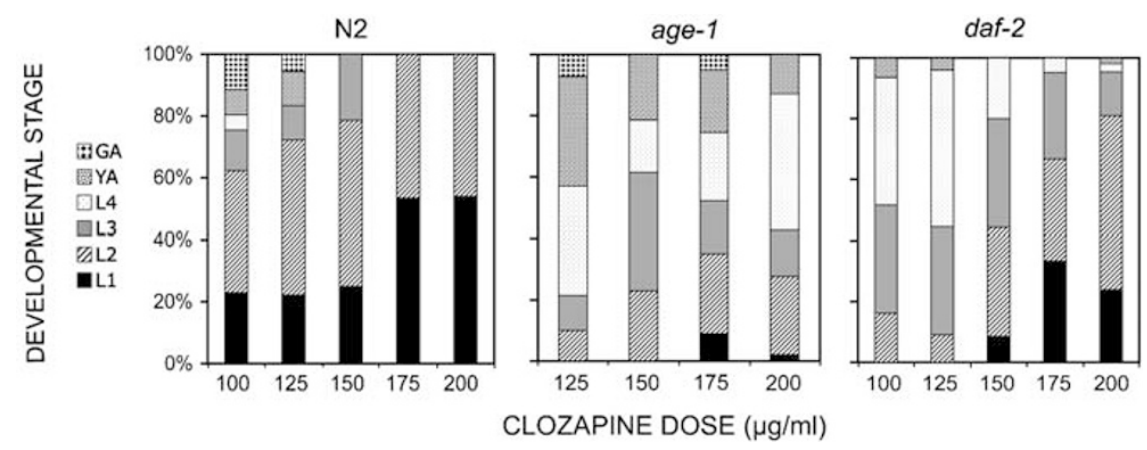

Figure 2 Larval arrest induced by clozapine is dose-dependent. The percentages of worms at different developmental stages are shown for the wild-type $\mathrm{N} 2$ worms and the mutants age-I (hx546) and daf-2(e/370) after 12 days of exposure at different dosages of clozapine. For age-I mutants, data for $100 \mu \mathrm{g} / \mathrm{ml}$ of clozapine are not shown because all animals had reached adulthood. 

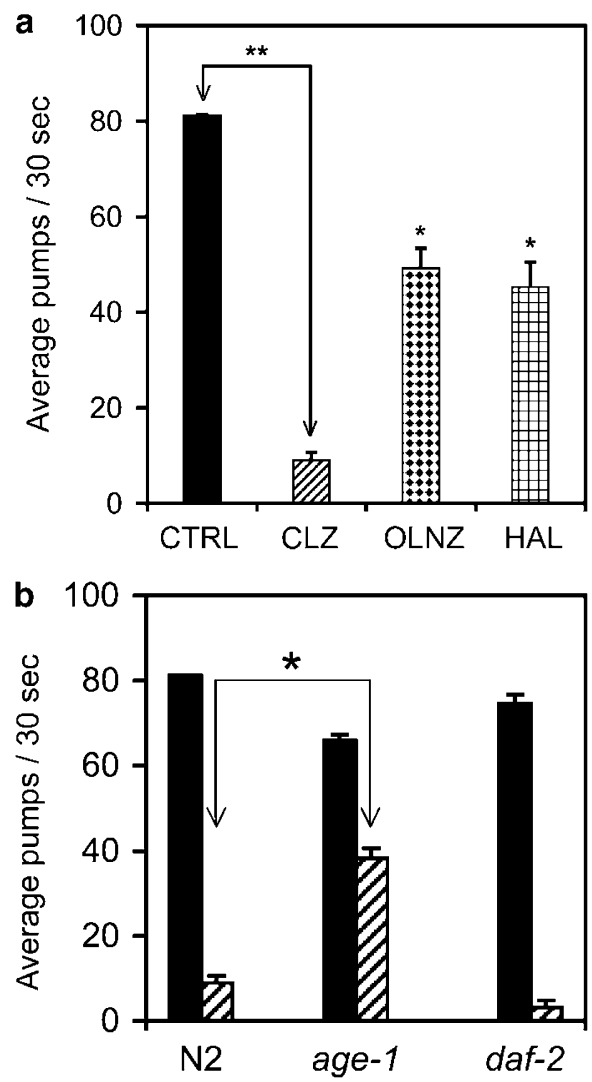

Figure 3 Effect of clozapine on pharyngeal pumping. (a) Pharyngeal pumping of wild-type LI larvae after exposure to $1 \%$ ethanol $(n=40)$, $200 \mu \mathrm{g} / \mathrm{ml}$ clozapine $(n=40), 50 \mu \mathrm{g} / \mathrm{ml}$ haloperidol $(n=20)$, and $100 \mu \mathrm{g} / \mathrm{ml}$ olanzapine $(n=20)$ for $24 \mathrm{~h}$. (b) Pharyngeal pumping of LI larvae in N2 $(n=90)$, age-l $(h \times 546)(n=40)$, and daf-2(e/370) $(n=20)$ worms after exposure to $1 \%$ ethanol and in N2 $(n=70)$, age- I $(h \times 546)(n=40)$, and daf-2(e / 370) $(n=20)$ worms after exposure to $200 \mu \mathrm{g} / \mathrm{ml}$ of clozapine for $24 \mathrm{~h}$. Error bars represent SEM. * and ** denote statistical significance.

pathway, a guanylate cyclase pathway, and a steroid hormone pathway. We tested mutations in the TGF- $\beta$ ligand $d a f-7$ and the TGF- $\beta$ receptor $d a f-4$, but saw no suppression of clozapine-induced larval arrest. We also tested the guanylate cyclase pathway mutants daf-11, tax-2, and tax-4 and the steroid hormone pathway mutant daf-9 (Supplementary Figure 4). None of these mutants suppressed clozapine-induced larval arrest. These studies suggest that clozapine acts through a limited number of specific pathways to effect larval arrest.

\section{Clozapine Inhibits Pharyngeal Pumping}

We tested whether clozapine produced larval arrest by inhibiting pharyngeal pumping in the larvae, thereby causing starvation. We found that exposure to clozapine resulted in a significant inhibition of pharyngeal pumping in N2 L1 larvae, much more pronounced than seen with haloperidol or olanzapine (Figure 3a). It should be noted that we had studied haloperidol at $50 \mu \mathrm{g} / \mathrm{ml}$ and olanzapine at $100 \mu \mathrm{g} / \mathrm{ml}$, their respective highest solubilities in ethanol, but we had used $200 \mu \mathrm{g} / \mathrm{ml}$ clozapine. To ensure that effects on pharyngeal pumping were not missed with haloperidol and olanzapine due to the lower dosage, we studied haloperidol and olanzapine at $200 \mu \mathrm{g} / \mathrm{ml}$ by dissolving them

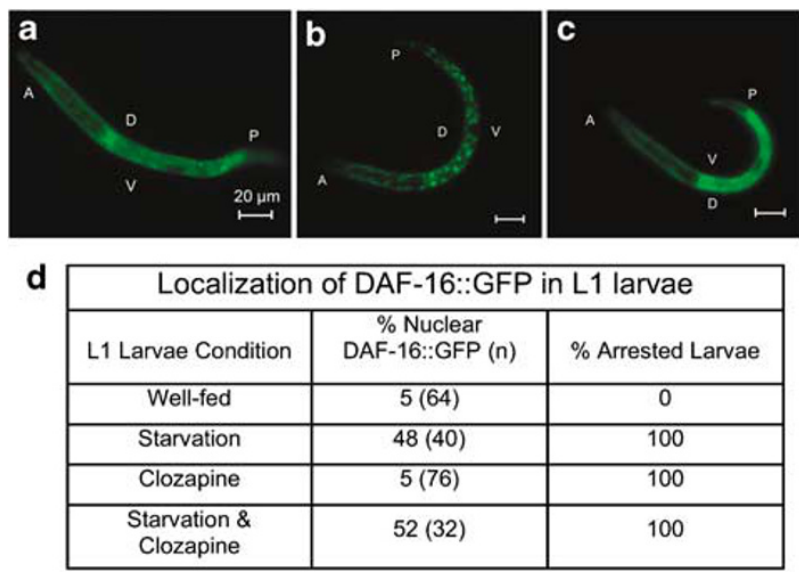

Figure 4 Clozapine and starvation have different effects on DAF-16 localization. Nuclear vs cytoplasmic localization of the fork head transcription factor DAF-16 in z/s356[DAF-16::GFP] worms, at $\times 200$ magnification. (a) Well-fed LI larvae show cytoplasmic localization of DAF-16::GFP. (b) Starvation-arrested LI larvae show nuclear accumulation of DAF-16:: GFP. (c) Clozapine-arrested LI larvae show cytoplasmic localization of DAF-16::GFP. (d) Quantification of nuclear DAF-16:: GFP levels.

in DMSO. Even at the higher concentrations, the degree of inhibition of pharyngeal pumping was several fold less than seen with clozapine. We then tested pharyngeal pumping in mutants to see if the same mutants that escaped larval arrest could also suppress clozapine-induced inhibition of pharyngeal pumping. We found that age-1 mutations partially rescued clozapine-induced inhibition of pharyngeal pumping in L1 larvae (Figure 3b). It is possible that decreased pharyngeal pumping contributes to the arrest seen with clozapine and that reversal of this defect may contribute to the suppression of arrest by age-1 mutants. However, daf-2 mutants, which partially rescue clozapine-induced larval arrest, do not rescue clozapine-induced inhibition of pharyngeal pumping in L1 larvae (Figure 3b). Hence, inhibition of pharyngeal pumping is not likely to be the sole reason for clozapine-induced larval arrest. We also sought to examine if eat mutants, which have reduced pharyngeal pumping at baseline, have increased sensitivity to clozapine. We performed dose-response experiments on two eat mutants, eat-7 (also known as egl-4) and eat-12 (also known as egl-19). Both of these strains display increased sensitivity to clozapine at an intermediate dose of $100 \mu \mathrm{g} / \mathrm{ml}$, consistent with the notion that clozapineinduced inhibition of pharyngeal pumping contributes to larval arrest (Supplementary Figure 5).

\section{Clozapine's Effects on the Insulin-Signaling Pathway}

One downstream effect of PI3K/AGE-1 in C. elegans is phosphorylation and inhibition of the FOXO transcription factor DAF-16 (Ogg et al, 1997; Mukhopadhyay et al, 2006). When $C$. elegans larvae are starved, the PI3K/AGE-1 pathway is inactivated, resulting in the nuclear translocation and activation of DAF-16. Such starvation leads to L1 arrest of the larvae (Baugh and Sternberg, 2006). By comparison, activation of the insulin-signaling pathway results in cytoplasmic localization of DAF-16. We undertook studies to see how clozapine affected the 

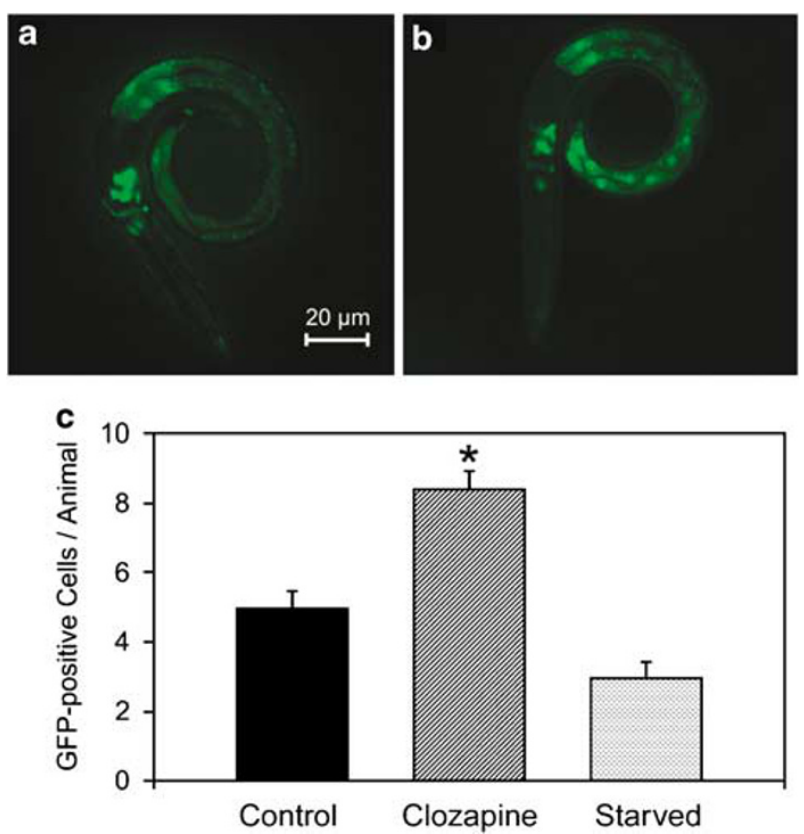

Figure 5 Clozapine induces AGE-I in intestinal cells. AGE-I::GFP expression in sEx 10837 (age- $1::$ GFP) worms under different conditions, at $\times 200$ magnification. (a) AGE-I ::GFP expression in LI larvae exposed to $1 \%$ ethanol for $24 \mathrm{~h}$. (b) AGE-I ::GFP expression in LI larvae exposed to $200 \mu \mathrm{g} / \mathrm{ml}$ of clozapine for $24 \mathrm{~h}$. (c) Quantification of AGE- I :: GFP-positive cells $(N=10$ for each condition). * denotes statistical significance.

nuclear/cytoplasmic localization of DAF-16, using worms containing an integrated daf-16:: GFP marker. Compared with starvation, exposure to clozapine elicited very different results. Although DAF-16 was localized in the nucleus in larvae arrested due to starvation, it was localized in the cytoplasm in larvae arrested due to clozapine exposure (Figure 4). Heat-shocked worms showed results identical to those observed with the starved larvae (data not shown). Our findings of cytoplasmic localization of DAF-16:: GFP in clozapine-arrested larvae indicates that clozapine is not inducing larval arrest by inhibiting the insulin-signaling pathway.

Cytoplasmic localization of DAF-16:: GFP in clozapinearrested larvae is consistent with the possibility that clozapine could be activating the insulin-signaling pathway. We tested whether exposure to clozapine induced the expression of AGE-1 by studying transgenic worms expressing AGE-1::GFP in the presence of clozapine. Clozapine exposure did not cause a change in AGE-1::GFP expression in the amphids and head neurons, but increased AGE$1::$ GFP signal in intestinal cells (Figure 5). This finding suggests that clozapine may induce the expression of AGE-1 in certain cellular contexts.

A recent study involving age-1 mutant suppressors revealed that there is more than one pathway for phospholipid-dependent kinases and that there are different separable larval and adult outputs of AGE-1 (Gami et al, 2006). We tested whether clozapine may influence a PI3K pathway distinct from the canonical PI3K-AKT-DAF-16 pathway. Consistent with this hypothesis, mutations in $p d k$ 1 and daf-16 did not affect clozapine-induced larval arrest. Similarly, neither an akt-1 mutant nor an akt-2 mutant rescued clozapine-induced larval arrest in our studies. We could not test an akt-1; akt-2 double mutant because akt-1; akt-2 double mutants result in dauer arrest, as is also seen in worms with concurrent RNAi inhibition of both akt-1 and $a k t-2$ (Paradis and Ruvkun, 1998; Hertweck et al, 2004; Oh et al, 2005).

In summary, we report a developmental phenotype of early L1-L2 larval arrest that is specific for clozapine vis-àvis other antipsychotics. Our studies suggest that clozapineinduced larval arrest is mediated by activation of the insulin-signaling pathway in C. elegans, because (1) mutations in age-1 and daf- 2 suppress clozapine-induced larval arrest; (2) clozapine produces cytoplasmic localization of DAF-16::GFP in arrested L1 larvae; and (3) clozapine induces increased expression of AGE-1::GFP.

\section{DISCUSSION}

\section{Clozapine Induces Larval Arrest in C. elegans}

Worms develop through four postembryonic larval stages before reaching adulthood (Riddle et al, 1997). Clozapine, but not other representative antipsychotic drugs including haloperidol, perphenazine and olanzapine, arrests worms in the early larval stages. In the presence of clozapine, wildtype embryos hatch but do not grow beyond L1-L2. Our results suggest that clozapine-induced larval arrest is not due to nonspecific toxicity, but is a specific effect on development that is exclusive to the L1 and L2 larval stages. Thus, the developmental effect of clozapine reported here is a fundamentally different phenomenon than the general slowing of growth reported with lower doses of antipsychotic drugs (Donohoe et al, 2006). The effect of $200 \mu \mathrm{g} / \mathrm{ml}$ clozapine is stage-specific and results in frank arrest, not just a delay in development.

C. elegans larvae arrest development as dauers when they encounter unfavorable conditions (Riddle et al, 1997). We tested the possibility that clozapine was activating the dauer pathway using recovery of dauer worms through selection with $1 \%$ SDS. Our results show that clozapine-induced larval arrest does not involve entry into the dauer stage. In addition, we tested animals with mutations in pathways that regulate entry into the dauer stage. Only mutants within the insulin-signaling pathway suppressed clozapine-induced larval arrest.

\section{Clozapine-Induced Larval Arrest is Suppressed by age-1 Mutants}

PI3K is well-conserved between mammals and C. elegans. DAF-2, the C. elegans homolog of insulin/IGF-1 receptors in humans, activates AGE-1/PI3K that transduces signals downstream to regulate growth and metabolism. In our study, mutant age-1 worms grew to adulthood in the presence of clozapine, whereas daf-2 mutations partially suppressed clozapine-induced larval arrest (Figure 2 and Supplementary Figure 3). As daf-2 mutants partially suppress clozapine-induced larval arrest but do not suppress clozapine-induced inhibition of pharyngeal pumping, suppression of clozapine-induced inhibition of pharyngeal pumping is not necessary for suppression of larval arrest. The finding that suppression of the pharyngeal pumping effect is not necessary for escape from 
clozapine-induced larval arrest does not exclude the possibility that decreased pharyngeal pumping may still contribute to clozapine-induced larval arrest. Inhibition of pharyngeal pumping and the resulting starvation are known to cause larval arrest. For example, mutants deficient in synaptic vesicle release, such as the synaptotagmin mutant snt-1, cause larval arrest by inhibition of pharyngeal pumping (Raizen et al, 1995). We find that clozapine causes an even more dramatic reduction of fluorescent bead uptake into the gut than snt-1 mutations (data not shown), though our DAF-16 localization results argue against starvation being the sole reason for clozapine-induced larval arrest. Clozapine may produce larval arrest through combined effects on parallel pathways, of which reduced pharyngeal pumping could be one.

The insulin-signaling pathway regulates dauer formation and aging in C. elegans. (Mukhopadhyay et al, 2006). Consistent with these findings, it has been reported that the insulin-signaling pathway specifically regulates L1 arrest induced by stressors such as starvation or high temperature (Baugh and Sternberg, 2006). It was shown that L1 larval arrest due to starvation is mediated by inhibition of the insulin-signaling pathway, resulting in activation and nuclear localization of the FOXO transcription factor DAF-16 (Baugh and Sternberg, 2006). However, in worms that undergo larval arrest during exposure to clozapine, DAF-16 is localized in the cytoplasm (Figure 4c). Activation of the insulin-signaling pathway drives DAF-16 into the cytoplasm, whereas starvation drives DAF-16 into the nucleus. Therefore, at least two of clozapine's biological effects compete with regard to DAF-16 localization. Activation of the insulin-signaling pathway may produce multiple effects, including inhibition of pharyngeal pumping. Alternatively, clozapine-induced activation of the insulin-signaling pathway and inhibition of pharyngeal pumping may occur in parallel. Importantly, we find that clozapine does not induce L1 arrest by simply acting as a stressor, because clozapine activates the insulin-signaling pathway, whereas stressors such as starvation or high temperature inactivate the pathway. The larval arrest reported here occurs in a biological context unique to the pharmacological effects of clozapine, a context that appears to involve parallel pathways.

\section{Implications for the Effects of Clozapine on Humans}

Given the conservation of the PI3K signaling pathways between $C$. elegans and humans, our results raise the possibility of an interaction between clozapine and PI3K pathways, including the insulin-signaling pathway, in mediating the effect(s) of clozapine in patients (Kalkman, 2006). Clozapine has uniquely superior therapeutic effects, even compared with the structurally similar antipsychotic olanzapine (Kumra et al, 2008). The molecular basis of clozapine's superior efficacy is not known. Although there has been much research focused on clozapine's effects on various neurotransmitter systems, there have been no definitive findings that explain clozapine's unique therapeutic and side effect profiles (Brunello et al, 1995). From a therapeutic standpoint, the potential for clozapine to modulate cerebral and peripheral glucose metabolism through the PI3K pathway would be of great clinical interest. Recent studies have pointed to a possible role of brain glucoregulatory processes in the pathophysiology of schizophrenia (Cohen et al, 1997; Chen et al, 2000; Clark et al, 2001; Fan et al, 2006; Holmes et al, 2006; Zhao et al, 2006; Venkatasubramanian et al, 2007). These studies had also suggested a unique effect of clozapine on cellular energy metabolism (Cohen et al, 1997). Our finding that clozapine alone among antipsychotic drugs tested induces larval arrest through the PI3K pathway may be mechanistically relevant in this regard.

A major side effect of clozapine is the induction of metabolic abnormalities and diabetes mellitus (Jin et al, 2002). The mechanism underlying the onset of diabetes is not clear (Wirshing et al, 1999). Interaction of clozapine with the PI3K pathway points to a possible mechanism for clozapine-induced metabolic effects. Indeed, the therapeutic effects and the metabolic side effects of clozapine may be related (Girgis et al, 2008). Although activation of the PI3K pathway in the brain may be therapeutic for psychiatric disorders, activation of the same pathway in other tissues may contribute to adverse metabolic effects. C. elegans has only one insulin receptor isoform but humans have two isoforms, HIR-A and HIR-B. The expression of these two isoforms varies by tissue - neurons express only HIR-A, liver expresses predominantly HIR-B, whereas adipose tissue and skeletal muscle express both isoforms (Sesti et al, 2001). By targeting HIR-A vis-à-vis HIR-B, it may be possible to develop therapeutic approaches that capitalize on the role of the insulin-signaling pathway in neurons while minimizing effects on other tissues that could be contributing to metabolic side effects. Alternatively, targeting the PI3K pathway downstream of the insulin receptor may have therapeutic value. In addition, discovery of clozapine's effect on developmental pathways may have implications for understanding clozapine's unique and problematic side effect of agranulocytosis. It has been shown that the clozapine metabolite $N$-desmethyl clozapine suppresses the development of hematopoietic precursors of myeloid and erythroid lineages (Gerson et al, 1994). Examination of clozapine's modulation of the PI3K pathway in the context of hematopoietic development would be worthwhile.

An interaction between clozapine and PI3K/AGE-1 is also interesting in the context of recent human genetic studies pointing to an association between AKT-1 and schizophrenia (Emamian et al, 2004; Arguello and Gogos, 2008). That study reported an association of schizophrenia with an AKT-1 haplotype that led to lower AKT-1 protein levels (Emamian et al, 2004). It has been observed that there is a decrease in AKT-1 levels in peripheral lymphocytes and brain of patients with schizophrenia (Emamian et al, 2004). Another study found that an AKT1 variant was associated with increased risk for schizophrenia and showed that AKT1 played a role in prefrontal-striatal structure and function in humans (Tan et al, 2008). These observations are also interesting in the context of reports of a decreased incidence of cancer in patients with schizophrenia as well as in their first-degree relatives (Lichtermann et al, 2001). Reports of a decrease in lung cancer incidence are especially remarkable in light of the high prevalence of cigarette smoking in patients with schizophrenia (Dervaux and Laqueille, 2008). PI3K-AKT-signaling pathway is one of the most commonly mutated systems in human cancers 
(Engelman et al, 2006). It is possible that patients with schizophrenia have decreased basal activity of the PI3KAKT signaling activity that makes them less susceptible to carcinogenesis, but more susceptible to deficits in the nervous system.

There are limitations to the experiments described here and to the generalization of results obtained in C. elegans to humans or other mammals. Identity of binding sites, either for drugs or neurotransmitters, has not been characterized in detail across species. Dosing of drugs by application in the media does not allow for tight control of drug levels in tissue and does not assure that these levels are similar to those attained in other organisms or model systems. The concentrations of drugs used in our studies were higher than those administered to humans, because high concentrations are required for penetration of the C. elegans cuticle. However, measurements of clozapine and $\mathrm{N}$-desmethyl clozapine in our study showed that the tissue levels are close to those expected in human brains. With these caveats, we report the finding of a clozapine-specific developmental arrest that is suppressed by mutations in PI $3 \mathrm{~K}$ and that may have implications for understanding the unique effects of clozapine and for the development of novel therapeutic approaches to psychosis.

As a result of these findings, we have initiated a genomewide RNA interference screen to identify and characterize other genes and pathways that are involved in clozapine's developmental effects in C. elegans. In addition, studies in rodent and other mammalian systems would be the next logical step. The ability to correlate PI3K effects of clozapine to behaviors or side effects will be complicated by the lack of validated rodent or other mammalian models for antipsychotic efficacy or side effects. An example of a possible follow-up study would be to determine if neuronspecific insulin receptor knockout mice show alterations in clozapine-induced behaviors such as facilitation of prepulse inhibition. In addition, one might study the effects of clozapine in surrogate tissues (ie, lymphocytes or fibroblasts) in patients with schizophrenia treated with clozapine. By studying PI3K activity in such tissues before and after clozapine treatment, one might be able to observe direct effects of clozapine on the PI3K pathway and could obtain data on how effects on PI3K activity correlate with the therapeutic or side effects of clozapine in patients. Such studies are beyond the scope of our experiments in $C$. elegans, but do illustrate how our findings in a nematode model system might lead to the identification of potentially important drug targets in other species.

\section{ACKNOWLEDGEMENTS}

The work was supported by the Adam Corneel Young Investigator Award, American Psychiatric Association/ Pfizer Research Fellowship, Harvard Medical School DuPont-Warren Fellowship and Livingston Fellowship to Rakesh Karmacharya, an NIH Clinical Scientist Development Award, a Bernhard Foundation grant and a NARSAD Young Investigator Award to Edgar A. Buttner, and a Stanley Medical Research Institute Center grant to Bruce M. Cohen. We thank Dr. Thomas Cooper at the Nathan Kline Institute in New York for assistance with the measurement of tissue clozapine levels, Dr. Mark Alkema at the University of Massachusetts, Worcester, MA, for assistance with integration of age-1::GFP, Lauraine Dalton for assistance with figures, and Theresa Stiernagle at the C. elegans Genetics Center for worm strains used in this work.

\section{DISCLOSURE/CONFLICT OF INTEREST}

R.F.S. is listed as a co-inventor on several patent applications with AstraZeneca, though no compensation has been received, nor anticipated in the near future, and the patent applications have no relevance to work presented in this paper. Beyond that, the authors declare that, except for income received from the primary employers, no financial support or compensation has been received from any individual or corporate entity over the past 3 years for research or professional service and there are no personal financial holdings that could be perceived as constituting a potential conflict of interest.

\section{REFERENCES}

Ambros V (2001). microRNAs: tiny regulators with great potential. Cell 28: 823-826.

Arguello PA, Gogos JA (2008). A signaling pathway AKTing up in schizophrenia. J Clin Invest 118: 2018-2021.

Arnt J, Skarsfeldt T (1998). Do novel antipsychotics have similar pharmacological characteristics? A review of the evidence. Neuropsychopharm 18: 63-101.

Baldessarini RJ, Centorrino F, Flood JG, Volpicelli SA, Huston Lyons D, Cohen BM (1993). Tissue concentrations of clozapine and its metabolites in the rat. Neuropsychopharm 9: 117-124.

Bargmann CI (1998). Neurobiology of the Caenorhabditis elegans genome. Science 282: 2028-2033.

Baugh LR, Sternberg PW (2006). DAF-16/FOXO regulates transcription of cki-1/Cip/Kip and repression of lin-4 during C. elegans L1 arrest. Curr Biol 16: 780-785.

Bettinger JC, Carnell L, Davies AG, McIntire SL (2004). The use of Caenorhabditis elegans in molecular neuropharmacology. Int Rev Neurobiol 62: 195-212.

Brar JS, Chengappa KN, Parepally H, Sandman AR, Kreinbrook SB, Sheth SA et al (1997). The effects of clozapine on negative symptoms in patients with schizophrenia with minimal positive symptoms. Ann Clin Psychiatry 9: 227-234.

Brunello N, Masotto C, Steardo L, Markstein R, Racagni G (1995). New insights into the biology of schizophrenia through the mechanism of action of clozapine. Neuropsychopharm 13: 177-213.

Carpenter WT, Koenig JI (2008). The evolution of drug development in schizophrenia: past issues and future opportunities. Neuropsychopharm 33: 2061-2079.

Centorrino F, Baldessarini RJ, Frankenburg FR, Kando J, Volpicelli SA, Flood JG (1996). Serum levels of clozapine and norclozapine in patients treated with selective serotonin reuptake inhibitors. Am J Psychiatry 153: 820-822.

Chen RY, Chen E, Ho WY (2000). A five-year longitudinal study of the regional cerebral metabolic changes of a schizophrenic patient from the first episode using Tc-99 m HMPAO SPECT. Eur Arch Psychiatry Clin Neurosci 250: 69-72.

Choy RK, Kemner JM, Thomas JH (2006). Fluoxetine-resistance genes in Caenorhabditis elegans function in the intestine and may act in drug transport. Genetics 172: 885-892.

Choy RK, Thomas JH (1999). Fluoxetine-resistant mutants in $C$. elegans define a novel family of transmembrane proteins. Mol Cell 3: 143-152. 
Clark C, Kopala L, Li DK, Hurwitz T (2001). Regional cerebral glucose metabolism in never-medicated patients with schizophrenia. Can J Psychiatry 46: 340-345.

Cohen RM, Nordahl TE, Semple WE, Andreason P, Litman RE, Pickar D (1997). The brain metabolic patterns of clozapineand fluphenazine-treated patients with schizophrenia during a continuous performance task. Arch Gen Psychiatry 54: 481-486.

Davies AG, Pierce-Shimomura JT, Kim H, VanHoven MK, Thiele TR, Bonci A et al (2003). A central role of the BK potassium channel in behavioral responses to ethanol in C. elegans. Cell 115: 655-666.

Davis JR, Li Y, Rankin CH (2008). Effects of developmental exposure to ethanol an Caenorhabditis elegans. Alcohol Clin Exp Res 32: 853-867.

Dervaux A, Laqueille X (2008). Smoking and schizophrenia: epidemiological and clinical features. Encephale 34: 299-305.

Donohoe DR, Aamodt EJ, Osborn E, Dwyer DS (2006). Antipsychotic drugs disrupt normal development in Caenorhabditis elegans via additional mechanisms besides dopamine and serotonin receptors. Pharm Res 54: 361-372.

Emamian ES, Hall D, Birnbaum MJ, Karayiorgou M, Gogos JA (2004). Convergent evidence for impaired AKT1-GSK3beta signaling in schizophrenia. Nat Genet 36: 131-137.

Engelman JA, Luo J, Cantley LC (2006). The evolution of phosphatidyl 3-kinases as regulators of growth and metabolism. Nat Rev Genet 7: 606-619.

Fan X, Liu E, Pristach C, Goff DC, Henderson DC (2006). Higher fasting serum insulin levels are associated with a better psychopathology profile in acutely ill non-diabetic inpatients with schizophrenia. Schiz Res 86: 30-35.

Fire A, Xu S, Montgomery MK, Kostas SA, Driver SE, Mello CC (1998). Potent and specific genetic interference by doublestranded RNA in Caenorhabditis elegans. Nature 391: 806-811.

Freedman R (2005). The choice of antipsychotic drugs for schizophrenia. N Engl J Med 353: 1286-1288.

Gami MS, Iser WB, Hanselman KB, Wolkow CA (2006). Activated AKT/PKB signaling in $C$. elegans uncouples temporally distinct outputs of DAF-2/insulin-like signaling. BMC Dev Biol 6: 45.

Gardner DM, Baldessarini RJ, Waraich P (2005). Modern antipsychotic drugs: a critical overview. Can Med Assoc J 172: 1703-1711.

Gerson SL, Arce C, Meltzer HY (1994). N-desmethylclozapine: a clozapine metabolite that suppresses haemopoiesis. $\mathrm{Br}$ J Haematol 86: 555-561.

Girgis RR, Javitch JA, Lieberman JA (2008). Antipsychotic drug mechanisms: links between therapeutic effects, metabolic side effects and the insulin signaling pathway. Mol Psychiatry 13: 918-929.

Hengartner MO, Horvitz HR (1994). Programmed cell death in Caenorhabditis elegans. Curr Opin Genet Dev 4: 581-586.

Hertweck M, Gobel C, Baumeister R (2004). C. elegans SGK-1 is the critical component in the Akt/PKB kinase complex to control stress response and life span. Dev Cell 6: 577-588.

Holmes E, Tsang TM, Huang JT-J, Leweke FM, Koethe D, Gerth CW et al (2006). Metabolic profiling of CSF: evidence that early intervention may impact on disease progression and outcome in schizophrenia. PLos Med 3: 1420-1428.

Horvitz HR (1999). Genetic control of programmed cell death in the nematode Caenorhabditis elegans. Cancer Res 59: 1701s-1706s.

Horvitz HR, Sternberg PW (1991). Multiple intercellular signalling systems control the development of the Caenorhabditis elegans vulva. Nature 351: 535-541.

Jin H, Meyer JM, Jeste DV (2002). Phenomenology of and risk factors for new-onset diabetes mellitus and diabetic ketoacidosis associated with atypical antipsychotics: an analysis of 45 published cases. Ann Clin Psychiatry 14: 59-64.
Kalkman HO (2006). The role of the phosphatidylinositide 3kinase-protein kinase B pathway in schizophrenia. Pharmacol Ther 110: 117-134.

Kane J, Honigfeld G, Singer J, Meltzer H (1988). Clozapine for the treatment-resistant schizophrenia. A double-blind comparison with chlorpromazine. Arch Gen Psychiatry 45: 789-796.

Krupp P, Barnes P (1992). Clozapine-associated agranulocytosis: risk and aetiology. Br J Psychiatry Suppl 17: 38-40.

Kumra S, Kranzler H, Gerbino-Rosen G, Kester HM, De Thomas C, Kafantaris V et al (2008). Clozapine and "high-dose" olanzapine in refractory early-onset schizophrenia: a 12-week randomized and double-blind comparison. Biol Psychiatry 63: 524-529.

Lamberti JS, Olson D, Crilly JF, Olivares T, Williams GC, Tu X et al (2006). Prevalence of the metabolic syndrome among patients receiving clozapine. Am J Psychiatry 163: 1273-1276.

Lee S, McIntire SL (2006). Chronic effects of ethanol in C. elegans. 16th International C. elegans meeting, 520B.

Lichtermann D, Ekelund J, Pukkala E, Tanskanen A, Lönnqvist J (2001). Incidence of cancer among persons with schizophrenia and their relatives. Arch Gen Psychiatry 58: 573-578.

Lieberman JA, Stroup TS, McEvoy JP, Swartz MS, Rosenheck RA, Perkins DO et al (2005). Effectiveness of antipsychotic drugs in patients with chronic schizophrenia. N Engl J Med 353: 1209-1223.

Lindenmayer JP, Iskander A, Park M, Apergi FS, Czobor P, Smith $\mathrm{R}$ et al (1998). Clinical and neurocognitive effects of clozapine and risperidone in treatment-refractory schizophrenic patients: a prospective study. J Clin Psychiatry 59: 521-527.

McKay SJ, Johnsen R, Khattra J, Asano J, Bailie DL, Chan S et al (2003). Gene expression profiling of cells, tissues, and developmental stages of the nematode C. elegans. Cold Spring Harb Symp Quant Biol 68: 159-170.

Meltzer HY, McGurk SR (1999). The effects of clozapine, risperidone, and olanzapine on cognitive function in schizophrenia. Schizophr Bull 25: 233-255.

Mukhopadhyay A, Oh SW, Tissenbaum HA (2006). Worming pathways to and from DAF-16/FOXO. Exp Gerontol 41: 928-934.

Ogg S, Paradis S, Gottlieb S, Patterson GI, Lee L, Tissenbaum HA et al (1997). The fork head transcription factor DAF-16 transduces insulin-like metabolic and longevity signals in $C$. elegans. Nature 389: 994-999.

Oh SW, Mukhopadhyay A, Svrzikapa N, Jiang F, Davis RJ, Tissenbaum HA (2005). JNK regulates lifespan in Caenorhabditis elegans by modulating nuclear translocation of forkhead transcription factor/DAF-16. Proc Natl Acad Sci USA 102: 4494-4499.

Paradis S, Ruvkun G (1998). Caenorhabditis elegans Akt/PKB transduces insulin receptor-like signals from AGE-1 PI3-kinase to the DAF-16 transcription factor. Genes Dev 12: 2488-2498.

Pierce SB, Costa M, Wisotzkey R, Devadhar S, Homburger SA, Buchman AR et al (2001). Regulation of DAF-2 receptor signaling by human insulin and ins-1, a member of the unusually large and diverse C. elegans insulin gene family. Genes Dev 15: 672-686.

Raizen DM, Lee RY, Avery L (1995). Interacting genes required for pharyngeal excitation by motor neuron MC in Caenorhabditis elegans. Genetics 141: 1365-1382.

Riddle DL, Blumenthal T, Meyer BJ, Priess JR (1997). C. elegans II. Cold Spring Harbor Laboratory Press, Cold Spring Harbor, NY.

Ruvkun G (2001). Glimpses of a tiny RNA world. Science 294: 797-799.

Sesti G, Federici M, Lauro D, Sbraccia P, Lauro R (2001). Molecular mechanism of insulin resistance in type 2 diabetes mellitus: role of the insulin receptor variant forms. Diabetes Metab Res Rev 17: 363-373.

Sieburth D, Ch'ng Q, Dybbs M, Tavazoie M, Kennedy S, Wang D et al (2005). Systematic analysis of genes required for synapse structure and function. Nature 436: 510-517. 
Sur C, Mallorga PJ, Wittmann M, Jacobsen MA, Pascarella D, Williams JB et al (2003). N-desmethylclozapine, an allosteric agonist at muscarinic 1 receptor, potentiates N-methyl-Daspartate receptor activity. Proc Natl Acad Sci USA 100: 13674-13679.

Tan HY, Nicodemus KK, Chen Q, Li Z, Brooke JK, Honea R et al (2008). Genetic variation in AKT1 is linked to dopamineassociated prefrontal cortical structure and function in humans. J Clin Invest 118: 2200-2208.

Venkatasubramanian G, Chittiprol S, Neelakantachar N, Naveen MN, Thirthall J, Gangadhar BB et al (2007). Insulin and insulin-like growth factor-1 abnormalities in antipsychotic-naive schizophrenia. Am J Psychiatry 164: 1557-1660.
Weiner DM, Meltzer HY, Veinbergs I, Donohue EM, Spalding TA, Smith TT et al (2004). The role of M1 muscarinic receptor agonism of $\mathrm{N}$-desmethylclozapine in the unique clinical effects of clozapine. Psychopharm (Berlin) 177: 207-216.

Weinshenker D, Garriga G, Thomas JH (1996). Genetic and pharmacological analysis of neurotransmitters controlling egg laying in C. elegans. J Neurosci 15: 6975-6985.

Wirshing DA, Wirshing WC, Kysar L, Berisford MA, Goldstein D, Pasdag J et al (1999). Novel antipsychotics: comparison of weight gain liabilities. J Clin Psychiatry 60: 358-363.

Zhao Z, Ksiezak-Reding H, Riggio S, Haroutunian V, Pasinetti GM (2006). Insulin receptor deficits in schizophrenia and in cellular and animal models of insulin receptor dysfunction. Schiz Res 84: $1-14$.

Supplementary Information accompanies the paper on the Neuropsychopharmacology website (http://www.nature.com/npp) 